\title{
Il campione parassitologico: appropriatezza pre-analitica
}

\section{*Marina Gaino **Giuseppe Tarabini Castellani}

*Laboratorio Microbiologia Ospedale S. Chiara Trento **Laboratorio Microbiologia Az. Osp. Pordenone

\section{INTRODUZIONE}

Il laboratorio di Microbiologia riveste un ruolo fondamentale nella diagnostica parassitologia (Tabella 1, 2 e 3 )

Per poter però avere la certezza della qualità del referto in una indagine parassitologia, come in qualsiasi altra indagine microbiologica, è necessario che le azioni alle quali il campione clinico viene sottoposto siano quelle appropriate. Le azioni analitiche alle quali deve essere sottoposto un campione possono essere divise in preanalitiche, analitiche e postanalitiche, noi ci preoccuperemo della prima fase cioè quella preanalitica. In

\section{Tabella I}

\begin{tabular}{|c|c|c|}
\hline \multicolumn{3}{|c|}{$\begin{array}{c}\text { RUOLO DEL LABORATORIO DI } \\
\text { MICROBIOLOGIA NELLA DIAGNOSTICA } \\
\text { PARASSITOLOGICA }\end{array}$} \\
\hline DIAG & $\begin{array}{l}\text { SI DELLE } \\
\text { ESTAZIOI } \\
\text { sostenut }\end{array}$ & $\frac{\mathrm{ONI}}{\mathrm{NNE}} \mathrm{ED}$ \\
\hline $\begin{array}{l}\text { parassiti } \\
\text { INTESTINALI }\end{array}$ & $\begin{array}{l}\text { parassiti } \\
\text { EMATICI }\end{array}$ & $\begin{array}{l}\text { parassiti di } \\
\text { altre SEDI } \\
\text { CORPOREE }\end{array}$ \\
\hline
\end{tabular}

Tabella 2

\section{CARATTERISTICHE DELL'ESAME PARASSITOLOGICO}

- importanza fondamentale dell'anamnesi geografica

- particolari modalità e tempi di raccolta del campione correlati al ciclo biologico del parassita
Tabella 3

\section{PARASSITOSI INTESTINALI}

- AUTOCTONE

- D'IMPORTAZIONE

viaggiatore immigrato bambino adottato

questa fase ci sono delle azioni che esulano dal laboratorio, ma che devono da esso essere indirizzate, come sono ad esempio la preparazione del paziente, le modalità di raccolta del campione ecc., e che vanno controllate all'arrivo del campione in laboratorio (Tabella 4).

\section{Tabella 4}

La qualita' della fase preanalitica è garantita da:

a) una raccolta appropriata del campione fecale

b) un adeguato numero di campioni fecali

c) l'idoneità degli eventuali conservanti utilizzati allo scopo

(per una fissazione ottimale il rapporto tra feci e fissativo deve essere 1:3)

Nelle tabelle successive (Tabella 5 e 6) si può osservare uno schema impostato secondo il tipo di campione e la sede di prelievo al quale applicare le metodiche di indagine atte al tipo di prelievo eseguito.

Noi ci limiteremo in questa sede a esporre le metodiche più comuni visive e colturali.

I campioni clinici per esame parassitologico possono provenire dall' apparato gastroenterico, urogenitale, respiratorio, dal sangue, dal sistema nervoso centrale, ottenuti direttamente oppure 
Tabella 5

\section{Campioni per esame parassitologico}

- Intestinali

- Uro-genitali

- Respiratori

- Ematici

- SNC

- Biopsie

Tabella 6

\section{Metodiche diagnostiche parassitologiche}

- Visive

- Colturali

mediante aspirazione o biopsia.

Le metodiche per l'indagine parassitologia classicamente adoperate sono quelle visive, nel caso che la parassitazione sia minima possono e debbono essere applicate metodiche colturali atte ad evidenziare i parassiti sospettati.

I campioni atti ad evidenziare parassiti del tratto gastroenterico possono pervenire, freschi o conservati in fissativo, sotto forma di feci spontaneamente emesse in modo naturale o provocate da purgante salino, oppure da aspirato duodenale, biliare o dal sigma colon. Per i parassiti intestinali endocellulari è necessario ricorrere alla biopsia. Le caratteristiche delle feci fresche per essere considerate appropriate per l'esame sono quelle di pervenire dopo l'emissione in meno di 30 minuti se sono liquide, entro 1 ora se si presentano molli o semiformate o entro le 12 ore se sono formate. Queste feci permettono la ricerca microscopica diretta a fresco dei trofozoiti mobili di Entamoeba histolytica, Giardia intestinalis, o la ricerca dei loro antigeni oppure la preparazioni di strisci per una colorazione. Non sono adeguati i campioni che presentino segni evidenti di essiccazione. Le feci conservate devono mantenere una proporzione $1: 3$ con il fissativo. Il numero "minimo"di campioni è 3 prelevati a giorni non consecutivi. Il fissativo deve essere consono alla metodica che si applicherà.

L'aspetto macroscopico delle feci, non applicabile a feci conservate, è utile per evidenziare sia la presenza di sangue o muco sulla superficie delle feci (presenza E. histolityca?), sia quella di ossiuri adulti o proglottidi di tenia. Lo stato di secchezza e/o l'età del campione oppure la presenza di sostanze estranee (bario? Feci colorate da rossiccio chiaro a bianco) possano inficiare le ricerche.

Le colorazioni permanenti, tricromica o ferro ematossilina, sono da eseguire su tutti i campioni, perché permettono una documentazione permanente, facilitano la identificazione di cisti e trofozoiti e permettono recuperare i piccoli trofozoiti persi con l'esame del concentrato a fresco.

Le procedure di concentrazione sono la centrifugazione delle feci filtrate e sgrasssate che è eseguibile con feci fresche oppure fissate in formalina (5-10\%), SAF(Sodio Acetato Formalina), MIF (Mertiolato Iodio Formalina), PVA (PoliVinyl Alcol), oppure la flottazione che non è applicabile a feci conservate in PVA. Per evidenziare coccidi o Ciclospora cayetanensis è necessario utilizzare colorazioni particolari come la Kinyoun o la Ziehl-Neelsen modificata, in questo caso è necessario che la ricerca sia indirizzata dal clinico. Queste colorazioni particolari sono applicabili sia a feci fresche che formalinate, a succo duodenale, bile, campioni respiratori ( espettorato indotto, prelievi bronchiali, campioni bioptici).

Per l'evidenziazione di nematodi (Ancylostomideae, Stongyloides spp., Trichostrongylus spp.) specie nei casi di scarsa parassitazione, può essere utile la coltura con i metodi di Harada-Mori, Baermann, o quello della carta bibula) da eseguire con feci fresche non fissate, non refrigerate nè congelate.

Campione sigmoideo.

Se è costituito da feci, muco o mucosa intestinale, il campione deve pervenire per la ricerca a fresco di trofozoiti mobili di Entamoeba histolytica, entro 30 minuti dal prelievo e mantenuto umido (su un letto assorbente (garza) inumidito con soluzione fisiologica a temperatura di $30-37^{\circ} \mathrm{C}$., oppure fissato o strisciato per la dimostrazione della presenza di protozoi.

Il contenuto duodenale ottenuto mediante Enterotest, oppure mediante aspirazione, è da considerarsi come feci fresche, è utile per la dimostrazione di larve di Strongyloides stercoralis, trofozoiti di G. intestinalis, oocisti di Cryptosporidium parvum, di Isospora belli e uova di Clonorchis sinensis.

I biopsiati intestinali sono utili per la dimostrazione di Microsporidi dopo colorazione di Giemsa o di Weber.

Schiusura delle uova di Schistosoma sp.: Questa tecnica sfrutta il fototropismo dei miracidi che fuoriescono dall'uovo dopo alcune ore di esposizione alla luce. Il campione deve essere fresco non conservato. E' una metodica applicabile sia a 
feci che a urine ed è utile nei parassitismo debole. Per la speciazione delle proglottidi di Tenia saginata o T. solium, le proglottidi devono pervenire fresche eventualmente mantenute in camera umida, ma non fissate. Per il recupero delle proglottidi e scolice di Tenia spp. Possono accettarsi feci fresche prima e dopo terapia, dopo purga salina dopo terapia e feci delle 24 ore formalinate (Tabelle 7, 8, 9 e 10).

\section{Tabella 7}

\section{RACCOLTA DEI CAMPIONI FECALI PER ESAME PARASSITOLOGICO}

- fornire al paziente istruzioni dettagliate sulle modalità di raccolta

- esaminare almeno 3 campioni raccolti a giorni alterni

- riportare su ogni campione data e ora di emissione

- allegare una richiesta con informazioni cliniche ed anamnestiche

\section{Tabella 8}

TRASPORTO e CONSERVAZIONE DEI CAMPIONI FECALI PER ESAME PARASSITOLOGICO

- il campione va esaminato appena giunto al laboratorio, oppure posto in flaconi contenenti sostanze conservanti o conservato a $4^{\circ} \mathrm{C}$

- campioni liquidi: esaminare entro $30 \mathrm{~min}$

- campioni non formati: esaminare entro $1 \mathrm{~h}$

- campioni formati: esaminare entro $12 \mathrm{~h}$

\section{Tabella 9}

\section{IL CAMPIONE PER ESAME COPRO PARASSITOLOGICO}

A) VERIFICARE LA CORRETTA ACCETTAZIONE ED IDENTIFICAZIONE DEI CAMPIONI

C) VERIFICARE LA CONGRUITA' TRA LA RICHIESTA E IL TIPO DI CAMPIONE

B) ESAMINARE MACROSCOPICAMENTE IL CAMPIONE FECALE PER:

- ADEguata QUANTITA'

CONSISTENZA DELLE FECI

PRESENZA DI MUCO O SANGUE

PRESENZA DI FORME PARASSITARIE

NON ACCETTARE CONTENITORI IMBRATTATI ALL'ESTERNO!
Tabella 10

\section{Parassitologico intestinale}

- Appropiatezza fissativo:

Sodio acetato formalina SAF: Colorazione permanente: SI

Formalina 5/10\%: $\quad$ Colorazione Permanente: NO

Polivinil alcol: $\quad$ Colorazione permanente: +/-

Mertiolato iodio formalina MIF: Colorazione permanente: NO

Ecofissativi:

Colorazione permanente: SI

Tutti permettono le procedure di concentrazione.

Il campione urogenitale, il cui prelievo ottimale dipende dal parassita ricercato, è costituito da urine o da secrezioni uretrali o vaginali spontanee oppure ottenute con ansa batteriologica, spazzolino coniforme o da un tampone di cotone o dacron. Questi si imbevono opportunamente nel secreto. I campioni devono pervenire in laboratorio entro 30 ' in camera umida o sospesi in 3-5 ml. di fisiologica a temperatura ambiente (TA) non refrigerati, per eseguire un esame diretto a fresco o colorato e seminarli negli opportuni terreni di coltura. La ricerca di Trichomonas vaginalis si eseguirà, nel maschio sulle prime goccie di urina del primo mitto mattutino con o senza massaggio della prostata, nella femmina, fondamentalmente nel secreto vaginale.

Per la ricerca delle Uova di Scistosoma haematobium, sono necessari $50 \mathrm{ml}$. di urine del mitto terminale meridiano, avvenendo il picco di escrezione tra le ore 12 e le 15.

Le urine: possono osservarsi per ricerca delle microfilarie, nei pazienti con chiluria, nelle infezioni ad elevata carica o in corso di terapia con dietilcarbamicina (DEC) nelle urine centrifugate (Tabella 11).

In un espettorato spontaneo possono riscontrarsi a fresco trofozoiti di Entamoeba histolytica, Uova di Paragonimus spp, larve di Strongyloides stercoralis, di Ancylostomidae, di Ascaris lumbricoides. Questi campioni devono pervenire al laboratorio entro 30' dall'emissione e con richiesta motivata.

In espettorato colorato con metodiche di ZiehlNeelsen o di Kinyoun, le stesse usate per l'evidenziazione di micobatteri, possiamo osservare oocisti di Cryptosporidium parvum.

Un espettorato indotto da vaporizzazioni con soluzione salina tiepida, oppure ottenuto mediante lavaggio broncoalveolare o spazzolato bronchiale sono i campioni indicati per la ricerca di Pneumocystis carinii (Tabella 12) 
Non possiamo dimenticare la parassitologia ematica, anche perché stanno aumentando le richieste dei colleghi clinici, ma soprattutto perché può impegnare la vita del paziente in tempi molto brevi e quindi va sempre considerato un esame urgente. Per la diagnosi di malaria, dovrebbero intercorrere non più di 60 minuti tra prelievo e risposta della lettura di uno striscio sottile.

Il campione clinico per parassitologia ematica

Tabella I I

\section{PARASSITI del TRATTO URO-GENITALE}

URINE

- uova di Schistosoma :

raccogliere almeno $10 \mathrm{ml}$ di urina del mitto terminale (tra le $\mathrm{h} 10 \mathrm{e}$ le h 14) in contenitore sterile; in alternativa raccogliere i mitti terminali delle $24 \mathrm{~h}$ (aggiungere formalina)

- Trichomonas vaginalis (nel maschio):

il pz. non deve aver urinato da circa $2 \mathrm{~h}$; raccogliere circa $10 \mathrm{ml}$ di urine del primo mitto; esaminare nel più breve tempo possibile (ev centrifugare) ed insemenzare il terreno di coltura

ESSUDATO VAGINALE e URETRALE

- Trichomonas vaginalis:

raccogliere il materiale e insemenzare il terreno di coltura

Tabella 12

\section{PARASSITI DELLE VIE RESPIRATORIE}

- ricerca oocisti di Cryptosporidium

- espettorato indotto

- lavaggio broncoalveolare

- occasionalmente: larve di elminti

(Ascaris 1., Strongyloides st., Ancylostoma /necator)

Tabella 13

\section{PARASSITI EMATICI E DEL SISTEMA RETICOLO-ENDOTELIALE}

Diagnosi di infezione da:

- Plasmodium spp.

- Leishmania spp.

- Trypanosoma spp.

Diagnosi di infestazione da Filarie costituito in genere da sangue circolante (a volte aspirato di midollo emopoietico) e tenuto fluido con anticoagulanti, permette la ricerca di plasmodi malarici, di babesie, tripanosomi, microfilaria e leishmania, sia su preparati a diretti fresco che arricchiti (goccia spessa, concentrazione per centrifugazione o filtrazione) o fissati e colorati, oppure la coltura in adeguati terreni. Il momento ottimale della raccolta del campione è, per i plasmodi, dopo 2 ore dall'inizio della ascesa febbrile. Per le microfilarie dipende dalla periodicità di esposizione al vettore, cioè per quelle di periodicità notturna - Wuchereria bancrofti, Brugia malayi-, dalle 22 alle 04 con il picco alle ore 24; per quelle a periodicità diurna- Loa loa- dalle 10 alle 16 con picco alle ore 13 . Per le aperiodiche Mansonella sp.- in qualsiasi momento dalle ore 0 alle ore 24 (Tabella 13).

Per la coltivazione dei parassiti, i campioni di feci (per E. hystolitica, S. stercoralis), sangue (per leishmanie e tripanosomi), secreti urogenitali, grattati corneali, liquido cefalo rachidiano devono arrivare in laboratorio entro 30 minuti dal prelievo o mantenuti a TA fino al momento della semina, che non deve avvenire oltre le 2 ore dal prelievo e non devono essere refrigerati.

\section{BIBLIOGRAFIA}

1. La corretta metodica dei prelievi. AA. Vari. 1982. 2. Manuali di servizio. Az Osp. Pn.

2. Diagnostic Medical Parasitology 2nd ed. Garcia L.S., Bruckner D.A. 1993. ASM. Washington, D.C.

3. Clinical microbiology procedures handbook. Isenerg, H.D., editor in chief: 1992 ASM. Washington, D.C.

4. Elementi di diagnostica parassitologica. AA. Vari. Quaderni di Microbiologia clinica. AMCLI .1994

5. De Carneri. Parassitologia generale e umana. 12.a ed.1997. Casa Editrice Ambrosiana Milano 